\title{
UAV and IoT Integration: A Flying Gateway
}

\author{
Aya Moheddine \\ University of Genoa \\ Genoa, Italy \\ aya.moheddine@edu.unige.it
}

\author{
Fabio Patrone \\ University of Genoa \\ Genoa, Italy \\ f.patrone@edu.unige.it
}

\author{
Mario Marchese \\ University of Genoa \\ Genoa, Italy \\ mario.marchese@unige.it
}

\begin{abstract}
This paper introduces a new approach for Internet of Things. This approach is based on the integration of IoT and Unmanned Aerial Vehicles (UAVs) to establish a flying gateway that allows the extension of coverage of terrestrial IoT gateways. The approach is based on using several hardware devices as Arduino, Raspberry Pi boards and RAK 2445 board offering LoRa connectivity. This LoRa-based gateway is deployed on board of a drone flying over IoT nodes to gather and transmit data to a LoRa server. This system will extend the coverage of the terrestrial LoRa gateways allowing to reach remote and rural areas.
\end{abstract}

Index Terms-UAV, IoT, Arduino, Rak2245, gateway

\section{INTRODUCTION}

Since the previous decades, Internet of Things (IoT) is witnessing an incredible growth. However, the past two years have pushed this field way far from expectations. It is expected to have about 76 billion IoT devices to be installed and connected worldwide in 2025 [1] (as shown in Figure 1). It is capturing a great interest in different sectors as industrial, agriculture, health-care, etc. Several technologies are established now allowing the communication between these devices and end users, some of which are considered as short-range as ZigBee, Bluetooth and $\mathrm{WiFi}$, and others are the long-range as SigFox and LoRa. Long-range transmission and low power consumption are the two main features that IoT communication protocols should respect. Long-range or LoRa is considered to be the most wireless radio frequency communication technology used in the IoT field. On the other hand, Unmanned Aerial Vehicles (UAVs) have been used since years, however they are witnessing an exceptional growth and high demand in the IoT area as Gartner mentioned 3 millions drones have been purchased in 2017 only [2]. Combining UAVs and IoT can lead to a great advantage specially in extending the coverage to rural and remote areas. UAVs have been used in so many fields such as military, natural disasters, crowd surveillance, real time monitoring, meteorology etc. Within the near future, UAVs will be used in different sectors as public safety, security and border surveillance, homeland security and goods transportation.

Several electronic devices, such as Arduino and Raspberry $\mathrm{Pi}$, can be employed as IoT gateways to allow the integration between different IoT devices and thus assembling the whole system. This paper will present a possible application of IoT devices, electronic components and drone to create our flying gateway.

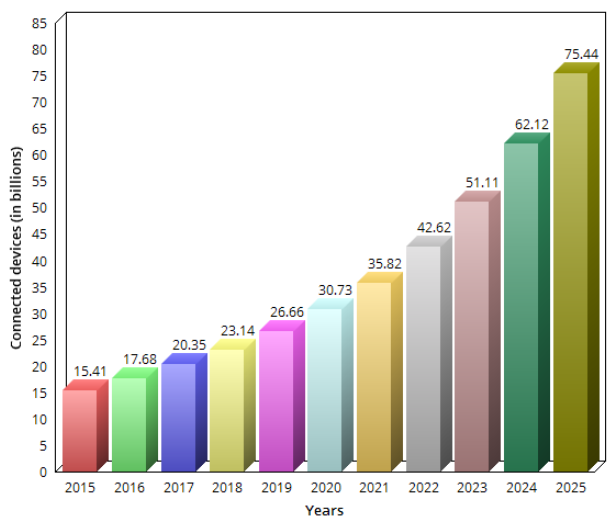

Fig. 1. An estimation of number of connected devices from 2015 till 2025 .

\section{RELATED WORK}

A crowd surveillance application composed of UAVs and IoT devices is presented in [3]. The used UAVs are equipped with video cameras and connected using (Long Term Evolution) LTE cellular networks to base stations. UAVs can be used along with IoT in evacuations systems. In [4] an evacuation system based on IoT and UAVs is given. This system is controlled by an agent that will determine the best evacuation plan based on the data collected from the sensors. Another integration of UAVs and IoT is in the agricultural domain which is transforming traditional farming systems to smart ones. Monitoring and controlling crops for improving the quality of food is presented in [5]. The different IoT devices are installed to sense the needed data and an UAV equipped with a light weight antenna is used to gather the data for analysis. Based on the state of art presented in this section, all the studies focus on having a UAV as an intermediate node allowing the collection and gathering of data generated by sensors, and transmitting them to the gateways ignoring its role as a flying gateway helping to reach remote areas. Our aim is to tackle this issue by designing a flying gateway to extend the coverage of terrestrial IoT gateways.

\section{PROPOSED APPROACH}

Figure 2 shows our proposed flying gateway system. In our approach the UAV will be the gateway, i.e., installing a LPWAN (Low Power Wide Area Network) gateway on board of the UAV. This allows the data to be transmitted to the gateway directly in remote areas which are out of coverage 
of terrestrial gateways. We use LoRa gateway because of the advantages it offers regarding long coverage and low power consumption and deploy it on board of a drone.

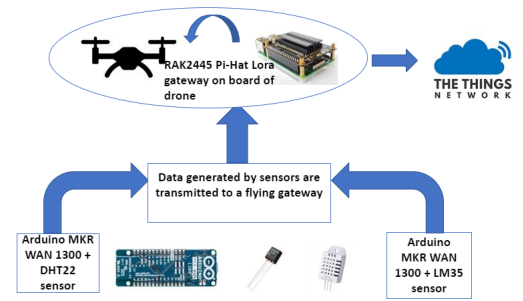

Fig. 2. Hardware architecture of the proposed approach.

\section{IMPLEMENTATION AND TESTING}

Our testbed is composed of two different IoT nodes based on two Arduino MKR WAN 1300 boards, one DHT22 and one LM35 temperature and humidity sensors and registered to The Things Network (TTN). Each IoT node is connected to a $868 \mathrm{MHz}$ antenna responsible of transmitting data to the gateway. With respect to the gateway, a Rak2245 Pi-Hat board is considered along with a Raspberry $\mathrm{Pi}$ as it provides LoRa connectivity with high coverage up to $15 \mathrm{~km}$ in open area. The RAK2245 Pi Hat is a module with Raspberry Pi form factor. This board can provide low data rate LoRa radio links in ultra-fast speed. It is powered by Semtech SX1301 transceiver concentrator capable of packet management from remotely spread nodes [6]. This gateway is registered on the server and the IoT nodes are connected to it. First the system is tested using Ethernet/WiFi connection, and as a second step using cellular network. For using cellular network, a Long Term Evolution (LTE) shield is placed on top of the Rak2245 board offering cellular connection. This gateway is then installed on a drone along with the power supply needed. The drone flies to the areas where the IoT nodes are installed offering extended coverage specially if they are installed in areas out of coverage of the cellular base stations. The gateway is able to receive the data collected from sensors and sends them using mobile connectivity to the server to be displayed and analyzed. Figure 3 shows our setup which is composed of two IoT nodes and the LoRa gateway. Each IoT node is made up of a sensor, Arduino MKR WAN 1300 board and an antenna. This setup is tested first using $\mathrm{Wi}-\mathrm{Fi}$, the results obtained shows no delay between the data transmission from sensors and the reception by the gateway up to distance 70 meters horizontally in a different room.

Graph represented in figure 4 shows the RSSI (Received Single Strength Indicator) values which represent the strength of the received signal and indicates how well the gateway can hear the signal from the IoT node. The small values of RSSI are because the packets were transmitted by IoT nodes near the gateway.

\section{CONCLUSION}

In this paper we present a new approach for the integration of UAVs in the IoT domain. Our aim is to design, install

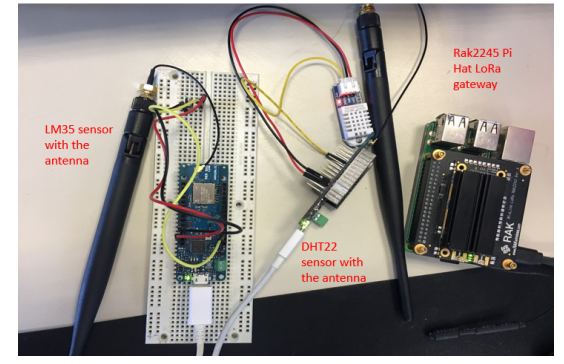

Fig. 3. Tested setup.

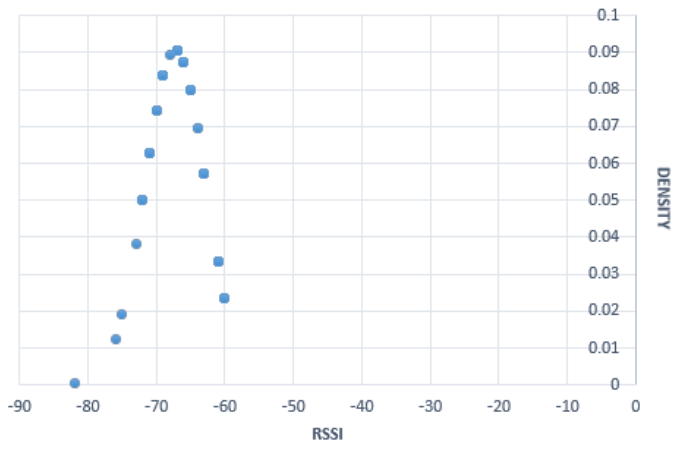

Fig. 4. Probability density function of RSSI values received by the gateway

and test a flying gateway system using Raspberry $\mathrm{Pi}$ and Rak2245 Pi-Hat boards, offering an opportunity for the users to reach IoT devices installed in remote areas out of the coverage of terrestrial gateways. Our future work will focus on integrating the above system with satellite communication as to extend more the coverage of LoRa network and offer a common solution to allow data exchange with multiple devices implementing different IoT communication protocols.

\section{REFERENCES}

[1] I. Ticlo, "Is Tech the New Currency? Why You Need Modern IT," https://www.insight.com/en_US/learn/content/2017/ 05252017-is-tech-the-new-currency-why-you-need-modern-it.html, 2017, [Online; accessed 17-September-2019].

[2] “,” https://www.gartner.com/en/newsroom/press-releases/ 2017-02-09-gartner-says-almost-3-million-personal-and-commercial-drones-will-be-sh 2017, [Online; accessed 19-September-2019].

[3] N. H. Motlagh, M. Bagaa, and T. Taleb, "Uav-based iot platform: A crowd surveillance use case," IEEE Communications Magazine, vol. 55, no. 2, pp. 128-134, February 2017.

[4] K. Katayama, H. Takahashi, S. Yokoyama, K. Gäfvert, and T. Kinoshita, "Evacuation guidance support using cooperative agent-based iot devices," in 2017 IEEE 6th Global Conference on Consumer Electronics (GCCE). IEEE, 2017, pp. 1-2.

[5] M. A. Uddin, A. Mansour, D. Le Jeune, and E. H. M. Aggoune, "Agriculture internet of things: Ag-iot," in 2017 27th International Telecommunication Networks and Applications Conference (ITNAC), Nov 2017, pp. 1-6.

[6] "RAK2245 Pi HAT Product Brief Description," https://downloads. rakwireless.com/en/LoRa/RAK2245-Pi-HAT/Hardware-Specification/ RAK2245_Pi_HAT_Product_Brief_V1.1.pdf, 2019, [Online; accessed 17-September-2019]. 